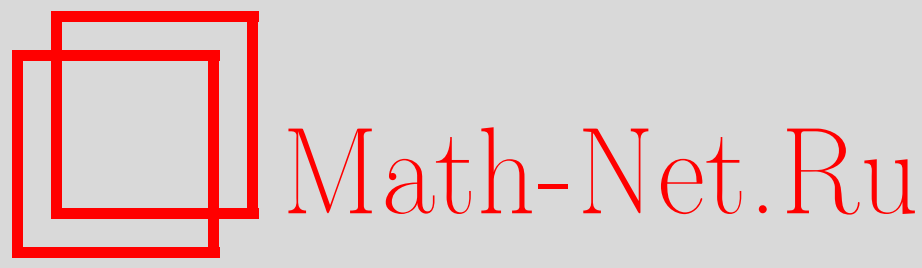

Хсу Чунгхао, Хань Дун, Асимптотическое поведение перколяционных кластеров с некоррелированными весами, ТМФ, 2008, том 157, номер 2, 309-320

DOI: https://doi.org/10.4213/tmf6281

Использование Общероссийского математического портала Math-Net.Ru подразумевает, что вы прочитали и согласны с пользовательским соглашением http://www . mathnet.ru/rus/agreement

Параметры загрузки:

IP : 3.89 .197 .203

26 апреля 2023 г., $16: 06: 28$

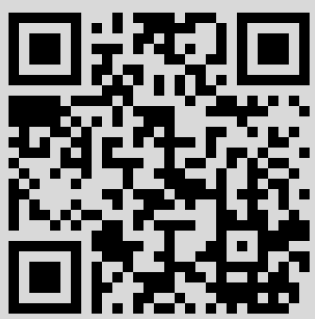




\section{АСИМПТОТИЧЕСКОЕ ПОВЕДЕНИЕ ПЕРКОЛЯЦИОННЫХ КЛАСТЕРОВ С НЕКОРРЕЛИРОВАННЫМИ ВЕСАМИ}

Рассматриваются случайные процессы, происходящие на реберных перколяционных кластерах и представляющие собой обобщение модели "подели и раскрась", введенной Хеггстремом в 2001 г. Исследуется асимптотическое поведение реберных перколяционных кластеров с некоррелированными весами. Для подкритических и надкритических фаз доказаны закон больших чисел и центральная предельная теорема в моделях соответственно так называемых quenched-вероятности и annealed-вероятности.

Ключевые слова: перколяционные кластеры, закон больших чисел, центральная предельная теорема.

\section{1. ВВЕДЕНИЕ И ОСНОВНЫЕ РЕЗУЛЬТАТЫ}

В последнее время не только многие специалисты в области теории вероятности, но и некоторые физики обратили свое внимание на динамическое поведение перколяционных кластеров. Основное внимание уделяется исследованию случайных процессов в перколяционных кластерах, но большая часть публикаций при этом посвящена исследованию случайных блужданий на надкритических перколяционных кластерах [1]-[4].

Цель данной работы состоит в исследовании кластерных моделей с весами, представляющих собой обобщение модели "подели и раскрась" (divide and color, DaC), введенной Хеггстремом [5]. DaC-модель легко описать: случайным образом выберем граф, отвечающий реберной перколяции, а затем раскрасим случайным образом и независимо различные кластеры (каждый кластер закрашивается одним и тем же цветом). Исследование такой модели раскрашенных кластеров необходимо с разных точек зрения. Наиболее важной оказывается связь с моделью Изинга [6], [7] и с моделью Поттса [8]. Во-первых, DaC-модель может быть использована как аль-

${ }^{*}$ Department of Mathematics, Shanghai Jiao Tong University, Shanghai, China. E-mail: hsuchunghao@gmail.com, donghan@sjtu.edu.cn 
тернатива моделям Изинга и Поттса при стохастическом моделировании различных пространственных систем с положительно коррелированными значениями в различных вершинах. Во-вторых, оказывается, что по сравнению с моделями Изинга и Поттса DaC-модель можно легко и непосредственно алгоритмизировать в виде двухступенчатой процедуры [5]. Гарет [9] провел дальнейшее исследование DaC-модели и изучил поведение намагниченности и флуктуаций при больших объемах решетки.

В настоящей работе исследуется асимптотическое поведение случайных графов с весами, в основном, для графов, случайно выбранных в соответствии с бернуллиевской реберной перколяцией на $\mathbb{Z}^{d}$. Выведены предельные теоремы для кластерных моделей с весами в случае так называемых quenched-вероятности и annealed-вероятности. Заметим, что подобные предельные результаты для моделей раскрашенных кластеров были получены Гаретом [9]. Однако доказательства в его работе были основаны на предположении, что цвета раскрасок для различных кластеров независимы и одинаково распределены (н.о.р.). Модель кластеров с весами, исследуемая в настоящей работе, имеет характер более общий, чем модель раскрашенных кластеров из работы [9], так как при н.о.р. цветах ослабляется условие некоррелированности раскрасок. Поэтому необходимо найти новые методы доказательств.

Прежде чем детально разработать модель кластеров с весами, напомним определение перколяции [10]. Зададим для ребер $e=\{x, y\} \in \mathbb{E}^{d}=\{\{x, y\}:|x-y|=1\}$ н.о.р. случайные бернуллиевские величины $\omega(e)$ с $\mathbb{P}_{p}(\omega(e)=1)=p \in[0,1]$, определенные на вероятностном пространстве $\left(\Omega, \mathcal{F}, \mathbb{P}_{p}\right)$, где $\Omega=\{0,1\}^{\mathbb{E}^{d}}$. Ребро е с $\omega(e)=1$ называется открытым, а открытый кластер $C(x)$, содержащий $x$, представляет собой множество $y$ таких, что $x$ и $y$ соединены открытым путем. Свойства этих так называемых перколяционных процессов широко исследуются. Хорошо известно, что существует критическое значение $p_{\mathrm{c}} \in(0,1)$ такое, что почти наверное

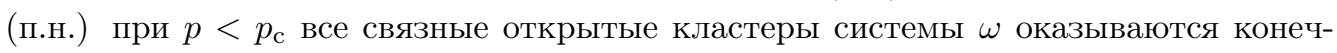

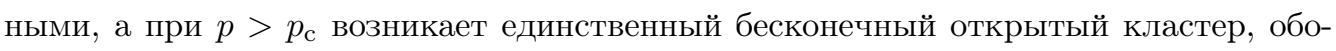
значаемый как $I=I(\omega)$. Относительно свойств перколяционных кластеров и более подробного описания теории перколяций см. [10]-[13].

Перколяционная конфигурация $\omega \in \Omega$ отождествляется с подграфом $\left(\mathbb{Z}^{d}, \mathbb{E}^{d}\right)$, множеством вершин которого является $\mathbb{Z}^{d}$, а множеством ребер $-\left\{e \in \mathbb{E}^{d}: \omega(e)=1\right\}$. Будем считать перколяционную конфигурацию случайной средой. При фиксированном $\omega \in \Omega$ будем считать $Y_{\omega}=\left\{X(x), x \in \mathbb{Z}^{d}\right\}$ случайным процессом на пространстве $\left(\mathbb{R}^{\mathbb{Z}^{d}}, \mathcal{B}\left(\mathbb{R}^{\mathbb{Z}^{d}}\right)\right)$, занумерованным $\mathbb{Z}^{d}$, где через $X(x)$ обозначен вес, соответствующий вершине $x \in \mathbb{Z}^{d}$. Случайные переменные $X(x)$ удовлетворяют следующим условиям:

$1)$ переменная $X(x)$ идентична переменной $X(y)$, если вершина $x$ связана с $y$;

2) переменные $X(x)$ и $X(y)$ некоррелированы, если $x$ не связана с $y$.

Обозначим через $P_{\omega}$ закон случайного процесса $Y_{\omega}$, который обычно называется quenched-вероятностью в фиксированной среде $\omega$. Зададим также annealed-вероятность $\mathbf{P}=\mathbb{P}_{p} \otimes P_{\omega}$. Через $E_{\omega}$ и $\mathbf{E}$ обозначим ожидания, взятые соответственно относительно вероятностей $P_{\omega}$ и $\mathbf{P}$. Условие 2 тогда можно переписать в таком виде: $E_{\omega} X(x) X(y)=E_{\omega} X(x) E_{\omega} X(y)$, если вершина $x$ не связана с вершиной $y$. 
В дальнейшем будем предполагать, что размерность $d \geqslant 2$ и что $\mu_{x}$ представляет собой закон распределения для $X(x)$ при всех $x \in \mathbb{Z}^{d}$. Без ограничения общности будем считать, что $\int X(x) d \mu_{x}=0$ при всех $x \in \mathbb{Z}^{d}$.

Пусть $B_{n}-$ ящик в $\mathbb{Z}^{d}$ с длинами сторон $2 n$ и центром в начале координат, т.е. $B_{n}=[-n, n]^{d} \cap \mathbb{Z}^{d}$. Для подмножества $A$ решетки $\mathbb{Z}^{d}$ обозначим через $|A|$ число вершин подмножества $A$.

Основными результатами данной работы являются следующие законы больших чисел и центральные предельные теоремы для quenched- и annealed-вероятностей.

ТЕОРема 1 (закон больших чисел для quenched-вероятности). 1. Подкритический режим при $p<p_{\text {с }}$. Для $\mathbb{P}_{p}$-почти всех $\omega \in \Omega \quad P_{\omega}$-n.н. имеет место сходимость

$$
\frac{\sum_{x \in B_{n}} X(x)}{\left|B_{n}\right|} \underset{n \rightarrow \infty}{\longrightarrow} 0 \text {. }
$$

2. Надкритический режим при $p>p_{\mathrm{c}}$. Для $\mathbb{P}_{p}$-почти всех $\omega \in \Omega \quad P_{\omega}$-n.н. имеет место сходимость

$$
\frac{\sum_{x \in B_{n}} X(x)}{\left|B_{n}\right|} \underset{n \rightarrow \infty}{\longrightarrow} \theta(p) Y,
$$

где $\theta(p)$ - вероятность того, что начало координат содержится в бесконечном кластере бернуллиевой перколячии с параметром $p$ из $\mathbb{Z}^{d}$, а $Y$ обозначает вес, присваиваемый бесконечному кластеру.

TЕорема 2 (закон больших чисел для annealed-вероятности). При $p \neq p_{\mathrm{c}} \mathbf{P}$-n.н. имеет место сходимость

$$
\frac{\sum_{x \in B_{n}} X(x)}{\left|B_{n}\right|} \underset{n \rightarrow \infty}{\longrightarrow} \theta(p) Y .
$$

ТЕорема 3 (центральная предельная теорема для quenched-вероятности). Предположим, что существуют положительные константы $C_{1} u C_{2}$ такие, что

$$
\int X^{4}(x) d \mu_{x} \leqslant C_{1}, \quad \sigma^{2}(x) \equiv \int X^{2}(x) d \mu_{x} \geqslant C_{2}
$$

при всех $x \in \mathbb{Z}^{d}$.

1. Подкритический режим. При $p<p_{\mathrm{c}}$ для $\mathbb{P}_{p}$-почти всех $\omega \in \Omega$ и при всех $t>0$

$$
P_{\omega}\left\{\frac{1}{\sqrt{\left|B_{n}\right|}} \sum_{x \in B_{n}} \frac{X(x)}{\sigma(x)} \leqslant t\right\} \underset{n \rightarrow \infty}{\longrightarrow} \frac{1}{\sqrt{2 \pi \chi^{f}(p)}} \int_{-\infty}^{t} \exp \left(-\frac{s^{2}}{2 \chi^{f}(p)}\right) d s
$$

где $\chi^{f}(p)$ - средний размер конечного кластера, m.e.

$$
\chi^{f}(p)=\sum_{k=1}^{\infty} k \mathbb{P}_{p}(|C(x)|=k)
$$


2. Надкритический режим. При $p>p_{\text {с }}$ для $\mathbb{P}_{p}$-почти всех $\omega \in \Omega$ и при всех $t>0$

$$
\begin{gathered}
P_{\omega}\left\{\frac{1}{\sqrt{\left|B_{n}\right|}}\left(\sum_{x \in B_{n} \backslash I} \frac{X(x)}{\sigma(x)}+\frac{1}{\sqrt{\left|B_{n} \cap I\right|}} \sum_{x \in B_{n} \cap I} \frac{X(x)}{\sigma(x)}\right) \leqslant t\right\} \underset{n \rightarrow \infty}{\longrightarrow} \\
\underset{n \rightarrow \infty}{\longrightarrow} \frac{1}{\sqrt{2 \pi\left(\chi^{f}(p)+\theta(p)\right)}} \int_{-\infty}^{t} \exp \left(-\frac{s^{2}}{2\left(\chi^{f}(p)+\theta(p)\right)}\right) d s,
\end{gathered}
$$

где I - бесконечный открытый кластер в перколяционной конфигурации $\omega$.

ТЕоремА 4 (центральная предельная теорема для annealed-вероятности). Предположим, что существуют положительные константы $C_{1} u C_{2}$ такие, что

$$
\int X^{4}(x) d \mu_{x} \leqslant C_{1}, \quad \sigma^{2}(x) \equiv \int X^{2}(x) d \mu_{x} \geqslant C_{2}
$$

при всех $x \in \mathbb{Z}^{d}$.

1. Подкритический режим. При $p<p_{\mathrm{c}}$ для всех $t>0$

$$
\mathbf{P}\left\{\frac{1}{\sqrt{\left|B_{n}\right|}} \sum_{x \in B_{n}} \frac{X(x)}{\sigma(x)} \leqslant t\right\} \underset{n \rightarrow \infty}{\longrightarrow} \frac{1}{\sqrt{2 \pi \chi^{f}(p)}} \int_{-\infty}^{t} \exp \left(-\frac{s^{2}}{2 \chi^{f}(p)}\right) d s .
$$

2. Надкритический режим. При $p>p_{\text {с }}$ для всех $t>0$

$$
\begin{gathered}
\mathbf{P}\left\{\frac{1}{\sqrt{\left|B_{n}\right|}}\left(\sum_{x \in B_{n} \backslash I} \frac{X(x)}{\sigma(x)}+\frac{1}{\sqrt{\left|B_{n} \cap I\right|}} \sum_{x \in B_{n} \cap I} \frac{X(x)}{\sigma(x)}\right) \leqslant t\right\} \underset{n \rightarrow \infty}{\longrightarrow} \\
\underset{n \rightarrow \infty}{\longrightarrow} \frac{1}{\sqrt{2 \pi\left(\chi^{f}(p)+\theta(p)\right)}} \int_{-\infty}^{t} \exp \left(-\frac{s^{2}}{2\left(\chi^{f}(p)+\theta(p)\right)}\right) d s .
\end{gathered}
$$

ЗАмечАниЕ 1. Теоремы 1-4 показывают, что на предельные поведения кластерных моделей с весами оказывают влияние свойства перколяционных процессов. Согласно законам больших чисел предел представляет собой константу в подкритическом режиме, но он не равен константе в надкритическом режиме. В центральных предельных теоремах пределы оказываются гауссовыми, но их параметры различны в двух указанных случаях.

\section{2. ПРЕДВАРИТЕЛЬНЫЕ РЕЗУЛЬТАТЫ}

Прежде чем перейти к доказательствам теорем, приведем несколько результатов перколяционной теории на ребрах.

Лемма 1. Предположим, что $p \neq p_{\mathrm{c}}$. Тогда существует $\alpha>0$ такое, что

$$
\mathbb{P}_{p}(n \leqslant|C(x)|<\infty) \leqslant e^{-\alpha n^{1 / d}}
$$


ДоКАЗАТЕЛЬСТВо. Подкритический случай следует из теоремы 3.4 работы [10], а надкритический случай - из формулы (6.94) работы [10] и теоремы 3 работы [13].

ЗАмечАниЕ 2. Лемма 1 показывает, что распределение размера кластера убывает экспоненциально как в подкритических, так и в надкритических фазах. Естественно, что интересно посмотреть на поведение кластерной модели в критической точке. В самом деле, предполагается, что существуют положительные константы $C_{1}, C_{2}$ и $\beta$ такие, что

$$
C_{1} n^{-(d-1) / 2} \leqslant \mathbb{P}_{p_{\mathrm{c}}}(n \leqslant|C(x)|<\infty) \leqslant C_{2} n^{-\beta} .
$$

Следующее утверждение доказано в работе [10] (теорема 4.2).

Лемма 2 [10]. Предположим, что $0 \leqslant p \leqslant 1$. Обозначим через $k(n)$ число открытых конечных кластеров, имеющих непустое пересечение с $B_{n}$, m.е.

$$
k(n)=\sum_{x \in B_{n}} \frac{1}{\left|C_{n}(x)\right|},
$$

где $C_{n}(x)$ - открытый кластер из $B_{n}$, содержащий $x$. Тогда для $\mathbb{P}_{p}$-почти всех $\omega \in \Omega$ число $k(n)$ удовлетворяет соотношению

$$
\frac{k(n)}{\left|B_{n}\right|} \underset{n \rightarrow \infty}{\longrightarrow} \kappa(p),
$$

где $\kappa(p)$ - число открытых кластеров на одну вершину, $\kappa(p)=\mathbb{E}_{p}|C(0)|^{-1}$.

Зададим $\gamma_{n}(x)$ при $x \in \mathbb{Z}^{d}$ следующим образом:

$$
\gamma_{n}(x)= \begin{cases}\left|C(x) \cap B_{n}\right|, & C(x)<\infty, \\ 0, & C(x)=\infty .\end{cases}
$$

Для $\omega \in \Omega$ предположим, что $\left\{x_{i} ; i \geqslant 1\right\}$ представляет собой последовательность, зависящую от $\omega$ и такую, что для каждой вершины $x \in \mathbb{Z}^{d}$ существует ровно одна вершина $x_{i}$, соединенная с $x$ в перколяционной конфигурации $\omega$. Иными словами, $x_{i}$ является вершиной-представителем в каждой связной компоненте. Тогда имеем

$$
\gamma_{n}\left(x_{i}\right)=0, \quad i>k(n) .
$$

Следующая лемма - следствие леммы 2 - доказана в работе [9]. Мы приводим ее доказательство для удобства дальнейшей работы.

Лемма 3. Предположим, что $p \neq p_{\mathrm{c}}$. Для $\mathbb{P}_{p}$-почти всех $\omega \in \Omega$ имеем

$$
\frac{\sum_{x \in B_{n}} \gamma_{n}(x)}{\left|B_{n}\right|} \underset{n \rightarrow \infty}{\longrightarrow} \chi^{f}(p) \text {. }
$$


ДокАЗАТЕЛЬСТво. Согласно лемме 1

$$
\begin{aligned}
\sum_{n=1}^{\infty} \mathbb{P}_{p}\left((\ln n)^{2 d}\right. & \leqslant|C(x)|<\infty) \leqslant \sum_{n=1}^{\infty} e^{-\alpha(\ln n)^{2}}=\sum_{n=1}^{\infty} n^{-\alpha \ln n}= \\
& =\sum_{n \leqslant e^{2 / \alpha}} n^{-\alpha \ln n}+\sum_{n>e^{2 / \alpha}} n^{-2}<\infty .
\end{aligned}
$$

По лемме Бореля-Кантелли $\mathbb{P}_{p}$-вероятность тех $\omega \in \Omega$, для которых существует положительная константа $N(\omega)$ такая, что $|C(x)| \leqslant(\ln n)^{2 d}$ при $n \geqslant N(\omega)$, равна единице. Тогда для $\mathbb{P}_{p}$-почти всех $\omega \in \Omega$ существует такое число $N(\omega)$, что $\gamma_{n}\left(x_{i}\right) \leqslant$ $(\ln n)^{2 d}$ для любого $i$ такого, что $1 \leqslant i \leqslant k(n)$, при всех $n \geqslant N$. Тем самым, имеем

$$
\sum_{\substack{x \in B_{n-(\ln n)^{2 d}}: \\|C(x)|<\infty}}|C(x)|=\sum_{\substack{x \in B_{n-(\ln n)^{2 d}}: \\|C(x)|<\infty}} \gamma_{n}(x) \leqslant \sum_{x \in B_{n}} \gamma_{n}(x) \leqslant \sum_{\substack{x \in B_{n}: \\|C(x)|<\infty}}|C(x)| .
$$

В силу теоремы об эргодичности (см. [14], теорема 6.1 и следствие 6.2$)$ для $\mathbb{P}_{p}$-почти всех $\omega \in \Omega$ получаем

$$
\frac{1}{\left|B_{n}\right|} \sum_{\substack{x \in B_{n}: \\|C(x)|<\infty}}|C(x)| \underset{n \rightarrow \infty}{\longrightarrow} \chi^{f}(p) .
$$

Результат леммы следует теперь из того, что

$$
\frac{\mid B_{n-(\ln n)^{2 d} \mid}}{\left|B_{n}\right|} \underset{n \rightarrow \infty}{\longrightarrow} 1 \text {. }
$$

Следующее предложение является ключевым при доказательстве теорем. Оно устанавливает закон больших чисел и центральную предельную теорему для двумерных массивов попарно некоррелированных случайных переменных.

ПрЕДЛОЖенИЕ. Пусть $\left\{X_{n}, n \geqslant 1\right\}$ - последовательность попарно некоррелированных (и одинаково распределенных) случайных переменных, заданных на вероятностном пространстве $(\widetilde{\Omega}, \widetilde{\mathcal{F}}, \widetilde{P}), \widetilde{E}$ - ожидание, соответствующее $\widetilde{P}, \sigma_{n}^{2}=$ $\widetilde{E}\left(X_{n}-\widetilde{E} X_{n}\right)^{2}, n \geqslant 1$. Пусть $\left\{a_{i j} ; i, j \geqslant 1\right\}-$ двухиндексная последовательность неотрицательных чисел, удовлетворяющая следующим условиям:

a) при всех $j \geqslant 1$ число $k(j)=\sup \left\{i \geqslant 1: a_{i j} \neq 0\right\}<\infty$ и существует константа $c>0$ такал, что $a_{i j} \geqslant c$, если $a_{i j} \neq 0$;

б) имеют место соотношения

$$
\sum_{j=1}^{\infty} \frac{1}{k(j)}<\infty, \quad \frac{1}{k(j)} \max _{1 \leqslant i \leqslant k(j)} a_{i j}^{4} \underset{j \rightarrow \infty}{\longrightarrow} 0
$$

в) при $j \rightarrow \infty$

$$
\frac{\sum_{i=1}^{\infty} a_{i j}^{2}}{\left(\sum_{i=1}^{\infty} a_{i j}\right)^{2}}=O\left(\frac{1}{k(j)}\right)
$$


Тогда справедливы следующие утверждения.

1. Закон больших чисел. Если существует константа $M>0$ такая, что $\sigma_{i}^{2} \leqslant$ при всех $i \geqslant 1$, то $\widetilde{P}-$ n.н.

$$
\frac{\sum_{i=1}^{\infty} a_{i j}\left(X_{i}-\widetilde{E} X_{i}\right)}{\sum_{i=1}^{\infty} a_{i j}} \underset{j \rightarrow \infty}{\longrightarrow} 0 .
$$

2. Центральная предельная теорема. Если существуют константы $M_{1}, M_{2}>0$ maкие, что $\widetilde{E}\left|X_{i}-\widetilde{E} X_{i}\right|^{4} \leqslant M_{1} u \sigma_{i}^{2} \geqslant M_{2}$ nри всех $i \geqslant 1$, mо при всех $t>0$

$$
\widetilde{P}\left\{\frac{\sum_{i=1}^{\infty} a_{i j}\left(X_{i}-\widetilde{E} X_{i}\right) / \sigma_{i}}{\sqrt{\sum_{i=1}^{\infty} a_{i j}^{2}}} \leqslant t\right\} \underset{j \rightarrow \infty}{\longrightarrow} \frac{1}{\sqrt{2 \pi}} \int_{-\infty}^{t} e^{-s^{2} / 2} d s .
$$

ДокАЗАТЕЛЬСТво. 1. Положим

$$
\eta_{j}=\frac{\sum_{i=1}^{\infty} a_{i j}\left(X_{i}-\widetilde{E} X_{i}\right)}{\sum_{i=1}^{\infty} a_{i j}}, \quad j \geqslant 1 .
$$

При $\varepsilon>0$ имеем

$$
\begin{aligned}
& \sum_{m=0}^{\infty} \widetilde{P}\left(\max _{2^{m+1}>n \geqslant 2^{m}}\left|\eta_{n}\right| \geqslant \varepsilon\right)= \\
& \quad=\widetilde{P}\left(\left|\eta_{1}\right| \geqslant \varepsilon\right)+\sum_{m=1}^{\infty} \widetilde{P}\left(\max _{2^{m+1}>n \geqslant 2^{m}}\left|\eta_{n}\right| \geqslant \varepsilon\right) \\
& \sum_{m=1}^{\infty} \widetilde{P}\left(\max _{2^{m+1}>n \geqslant 2^{m}}\left|\eta_{n}\right| \geqslant \varepsilon\right)=\sum_{m=1}^{\infty} \widetilde{P}\left(\bigcup_{n=2^{m}}^{2^{m+1}-1}\left\{\left|\eta_{n}\right| \geqslant \varepsilon\right\}\right) \leqslant \\
& \leqslant \sum_{m=1}^{\infty} \sum_{n=2^{m}}^{2^{m+1}-1} \widetilde{P}\left\{\left|\eta_{n}\right| \geqslant \varepsilon\right\} \leqslant \sum_{m=1}^{\infty} \sum_{n=2^{m}}^{2^{m+1}-1} \frac{\widetilde{E}\left(\eta_{n}-\widetilde{E} \eta_{n}\right)^{2}}{\varepsilon^{2}}= \\
& \quad=\sum_{m=1}^{\infty} \sum_{n=2^{m}}^{2^{m+1}-1} \frac{\sum_{i=1}^{k(n)} a_{i n}^{2} \sigma_{i}^{2}}{\varepsilon^{2}\left(\sum_{i=1}^{k(n)} a_{i n}\right)^{2}} \leqslant \frac{M}{\varepsilon^{2}} \sum_{n=2}^{\infty} \frac{C}{k(n)}<\infty
\end{aligned}
$$

Таким образом, при $\varepsilon>0$ получаем

$$
\sum_{m=0}^{\infty} \widetilde{P}\left(\max _{2^{m+1}>n \geqslant 2^{m}}\left|\eta_{n}\right| \geqslant \varepsilon\right)<\infty .
$$

В соответствии с леммой Бореля-Кантелли имеем

$$
\widetilde{P}\left(\max _{2^{m+1}>n \geqslant 2^{m}}\left|\eta_{n}\right| \geqslant \varepsilon \text { i.o. }\right)=0,
$$

(здесь и далее “і.о.” означает, что имеет место бесконечное по $m$ число событий под знаком вероятности), т.е.

$$
\widetilde{P}\left(\bigcup_{n=2^{m}}^{2^{m+1}-1}\left\{\left|\eta_{n}\right| \geqslant \varepsilon\right\} \text { i.o. }\right)=0
$$


Учитывая, что

$$
\begin{aligned}
\left\{\left|\eta_{n}\right| \geqslant \varepsilon \text { i.o. }\right\} & =\bigcap_{k=1}^{\infty} \bigcup_{n=k}^{\infty}\left\{\left|\eta_{n}\right| \geqslant \varepsilon\right\}=\bigcap_{k=1}^{\infty} \bigcup_{m=k}^{\infty} \bigcup_{n=2^{m}}^{2^{m+1}-1}\left\{\left|\eta_{n}\right| \geqslant \varepsilon\right\}= \\
& =\left\{\bigcup_{n=2^{m}}^{2^{m+1}-1}\left\{\left|\eta_{n}\right| \geqslant \varepsilon\right\} \text { i.o. }\right\},
\end{aligned}
$$

получаем

$$
\widetilde{P}\left(\left|\eta_{n}\right| \geqslant \varepsilon \text { i.o. }\right)=0 .
$$

Отсюда следует, что $\widetilde{P}$-п.н. имеет место сходимость $\eta_{n} \underset{n \rightarrow \infty}{\longrightarrow} 0$.

2. Положим

$$
W_{j}=\frac{\sum_{i=1}^{\infty} a_{i j}\left(X_{i}-\widetilde{E} X_{i}\right) / \sigma_{i}}{\sqrt{\sum_{i=1}^{\infty} a_{i j}^{2}}} .
$$

Тогда $\widetilde{E} W_{j}=0$ и $\widetilde{E}\left[W_{j}^{2}\right]=1$. Применим теперь теорему 2.4 работы [15]. Заметим, что доказательство указанной теоремы останется справедливым, если заменить каждый термин независимый термином некоррелированный в определении графа зависимостей. Таким образом, достаточно показать, что

$$
\sum_{i=1}^{\infty} \widetilde{E}\left|\frac{a_{i j}\left(X_{i}-\widetilde{E} X_{i}\right) / \sigma_{i}}{\sqrt{\sum_{i=1}^{\infty} a_{i j}^{2}}}\right|^{4} \underset{j \rightarrow \infty}{\longrightarrow} 0
$$

В самом деле,

$$
\sum_{i=1}^{\infty} \widetilde{E}\left|\frac{a_{i j}\left(X_{i}-\widetilde{E} X_{i}\right) / \sigma_{i}}{\sqrt{\sum_{i=1}^{\infty} a_{i j}^{2}}}\right|^{4} \leqslant \frac{\sum_{i=1}^{k(j)} a_{i j}^{4} M_{1}}{\left(\sum_{i=1}^{k(j)} a_{i j}^{2}\right)^{2} M_{2}} \leqslant \widetilde{C} \frac{k(j) \max _{1 \leqslant i \leqslant k(j)} a_{i j}^{4}}{k(j)^{2}} \underset{j \rightarrow \infty}{\longrightarrow} 0
$$

где второе неравенство следует из условий "а" и "в", а последнее соотношение вытекает из условия "б".

\section{3. ДОКАЗАТЕЛЬСТВА ТЕОРЕМ}

\section{1. Закон больших чисел.}

ДокАЗАТЕЛЬСтво теоремы 1. Докажем теорему сразу в подкритическом и надкритическом режимах. Имеем

$$
\begin{aligned}
\sum_{x \in B_{n}} X(x) & =\sum_{x \in B_{n} \backslash I} X(x)+\sum_{x \in B_{n} \cap I} X(x)= \\
& =\sum_{i=1}^{\infty} \gamma_{n}\left(x_{i}\right) X\left(x_{i}\right)+Y\left|B_{n} \cap I\right|,
\end{aligned}
$$

где $I$ - бесконечная компонента, если такая существует, $Y$ - ее вес. 
Пусть $a_{i j}=\gamma_{j}\left(x_{i}\right)$. Применим п. 1 доказанного предложения к последовательности $\left\{a_{i j} ; i, j \geqslant 1\right\}$ и к случайным переменным $\left\{X\left(x_{i}\right) ; i \geqslant 1\right\}$. Заметим, что

$$
\sum_{i=1}^{\infty} \gamma_{n}\left(x_{i}\right)=\left|B_{n} \backslash I\right|=\sum_{x \in B_{n}} 1_{\{C(x)<\infty\}}
$$

Учитывая равенство

$$
\left|B_{n} \cap I\right|=\sum_{x \in B_{n}} 1_{\{C(x)=\infty\}}
$$

и теоремы эргодичности (см. [14], теорема 6.1 и следствие 6.2), имеем

$$
\lim _{n \rightarrow \infty} \frac{\left|B_{n} \backslash I\right|}{\left|B_{n}\right|}=1-\theta(p), \quad \lim _{n \rightarrow \infty} \frac{\left|B_{n} \cap I\right|}{\left|B_{n}\right|}=\theta(p) .
$$

Тогда в соответствии с п. 1 предложения получаем, что $P_{\omega}$-п.н.

$$
\frac{\sum_{i=1}^{\infty} \gamma_{n}\left(x_{i}\right) X\left(x_{i}\right)}{\left|B_{n} \backslash I\right|} \underset{n \rightarrow \infty}{\longrightarrow} 0 .
$$

Таким образом,

$$
\begin{aligned}
\frac{\sum_{x \in B_{n}} X(x)}{\left|B_{n}\right|} & =\frac{\left|B_{n} \backslash I\right|}{\left|B_{n}\right|} \frac{\sum_{x \in B_{n} \backslash I} X(x)}{\left|B_{n} \backslash I\right|}+\frac{\sum_{x \in B_{n} \cap I} X(x)}{\left|B_{n}\right|}= \\
& =\frac{\left|B_{n} \backslash I\right|}{\left|B_{n}\right|} \frac{\sum_{i=1}^{\infty} \gamma_{n}\left(x_{i}\right) X\left(x_{i}\right)}{\left|B_{n} \backslash I\right|}+\frac{Y\left|B_{n} \cap I\right|}{\left|B_{n}\right|} \underset{n \rightarrow \infty}{\longrightarrow} \theta(p) Y \quad \text { (P } P_{\omega} \text {-П.н.). }
\end{aligned}
$$

Теорема 1 доказана.

ЗАмЕЧАниЕ 3. Предложение 2.1 из [5] можно вывести из теоремы 1.

ДокАЗАТЕЛьство теоремы 2. Для случая annealed-вероятности утверждение следует из результата для quenched-вероятности. Согласно теореме 1 имеем $P_{\omega}(\Xi)=1$, где

$$
\Xi=\left\{\frac{\sum_{x \in B_{n}} X(x)}{\left|B_{n}\right|} \underset{n \rightarrow \infty}{\longrightarrow} \theta(p) Y\right\}
$$

Таким образом,

$$
\mathbf{P}(\Xi)=\int_{\Omega} P_{\omega}(\Xi) \mathbb{P}_{p}(d \omega)=\int_{\Omega} 1 \mathbb{P}_{p}(d \omega)=1 .
$$

\section{2. Центральные предельные теоремы.}

ДокАЗАТЕЛЬСТВо тЕОРЕмы 3. Для доказательства центральной предельной теоремы приме́ним п. 2 предложения.

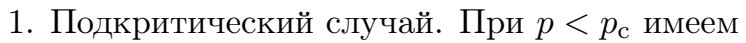

$$
\sum_{x \in B_{n}} \frac{X(x)}{\sigma(x)}=\sum_{i=1}^{\infty} \gamma_{n}\left(x_{i}\right) \frac{X\left(x_{i}\right)}{\sigma\left(x_{i}\right)} .
$$


Заметим, что

$$
\gamma_{n}\left(x_{i}\right)\left\{\begin{array}{lll}
=0, & \text { если } & i>k(n), \\
\geqslant 1, & \text { если } \quad i \leqslant k(n)
\end{array}\right.
$$

и в силу леммы 2 получаем

$$
k(n) \sim \kappa(p)\left|B_{n}\right|=\kappa(p)(2 n+1)^{d} .
$$

Таким образом,

$$
\sum_{n=1}^{\infty} \frac{1}{k(n)}<\infty
$$

Из доказательства леммы 3 вытекает, что для $\mathbb{P}_{p}$-почти всех $\omega \in \Omega$ существует число $N$ такое, что при всех $n \geqslant N$ и для любого $i, 1 \leqslant i \leqslant k(n)$, справедливо неравенство $\gamma_{n}\left(x_{i}\right) \leqslant(\ln n)^{2 d}$. Следовательно, в силу леммы 2

$$
\frac{1}{k(n)} \max _{1 \leqslant i \leqslant k(n)} \gamma_{n}^{4}\left(x_{i}\right)=\frac{1}{\left|B_{n}\right|} \max _{1 \leqslant i \leqslant k(n)} \gamma_{n}^{4}\left(x_{i}\right) \frac{\left|B_{n}\right|}{k(n)} \underset{n \rightarrow \infty}{\longrightarrow} 0
$$

Более того, имеем

$$
\begin{aligned}
\frac{\sum_{i=1}^{\infty} \gamma_{i n}^{2}}{\left(\sum_{i=1}^{\infty} \gamma_{i n}\right)^{2}} k(n) & =\frac{\sum_{i=1}^{k(n)} \gamma_{i n}^{2}}{\left(\sum_{i=1}^{k(n)} \gamma_{i n}\right)^{2}} k(n)=\frac{\sum_{x \in B_{n}} \gamma_{n}(x)}{\left|B_{n}\right|^{2}} k(n)= \\
& =\frac{\sum_{x \in B_{n}} \gamma_{n}(x)}{\left|B_{n}\right|} \frac{k(n)}{\left|B_{n}\right|} \underset{n \rightarrow \infty}{\longrightarrow} \chi^{f}(p) \kappa(p) .
\end{aligned}
$$

Тогда при $n \rightarrow \infty$

$$
\frac{\sum_{i=1}^{\infty} \gamma_{i n}^{2}}{\left(\sum_{i=1}^{\infty} \gamma_{i n}\right)^{2}}=O\left(\frac{1}{k(n)}\right)
$$

Так как

$$
\begin{aligned}
\frac{\sum_{x \in B_{n}} \frac{X(x)}{\sigma(x)}}{\sqrt{\left|B_{n}\right|}}= & \sqrt{\frac{\sum_{i=1}^{\infty} \gamma_{n}^{2}\left(x_{i}\right)}{\left|B_{n}\right|} \frac{\sum_{i=1}^{\infty} \gamma_{n}\left(x_{i}\right) X\left(x_{i}\right) / \sigma\left(x_{i}\right)}{\sqrt{\sum_{i=1}^{\infty} \gamma_{n}^{2}\left(x_{i}\right)}}}, \\
& \sum_{i=1}^{\infty} \gamma_{n}^{2}\left(x_{i}\right)=\sum_{x \in B_{n}} \gamma_{n}(x),
\end{aligned}
$$

первое утверждение теоремы следует из леммы 3 и п. 2 предложения.

2. Надкритический случай. Сначала заметим, что

$$
\frac{1}{\sqrt{\left|B_{n}\right|}} \sum_{x \in B_{n} \backslash I} \frac{X(x)}{\sigma(x)}=\frac{1}{\sqrt{\left|B_{n}\right|}} \sum_{i=1}^{\infty} \gamma_{n}\left(x_{i}\right) \frac{X\left(x_{i}\right)}{\sigma\left(x_{i}\right)} .
$$


Выберем вершину $x_{0} \in B_{n} \cap I$ и определим $\gamma_{n}\left(x_{0}\right)=\sqrt{\left|B_{n} \cap I\right|}$. Тогда

$$
\begin{aligned}
& \frac{1}{\sqrt{\left|B_{n}\right|}}\left(\sum_{x \in B_{n} \backslash I} \frac{X(x)}{\sigma(x)}+\frac{1}{\sqrt{\left|B_{n} \cap I\right|}} \sum_{x \in B_{n} \cap I} \frac{X(x)}{\sigma(x)}\right)=\frac{\sum_{i=0}^{k(n)} \gamma_{n}\left(x_{i}\right) X\left(x_{i}\right) / \sigma\left(x_{i}\right)}{\sqrt{\left|B_{n}\right|}}= \\
& =\frac{\sqrt{\sum_{i=0}^{k(n)} \gamma_{n}^{2}\left(x_{i}\right)}}{\sqrt{\left|B_{n}\right|}} \frac{\sum_{i=0}^{k(n)} \gamma_{n}\left(x_{i}\right) X\left(x_{i}\right) / \sigma\left(x_{i}\right)}{\sqrt{\sum_{i=0}^{k(n)} \gamma_{n}^{2}\left(x_{i}\right)}} .
\end{aligned}
$$

С одной стороны,

$$
\begin{aligned}
\frac{\sum_{i=0}^{k(n)} \gamma_{n}^{2}\left(x_{i}\right)}{\left|B_{n}\right|} & =\frac{\sum_{i=1}^{k(n)} \gamma_{n}^{2}\left(x_{i}\right)}{\left|B_{n}\right|}+\frac{\gamma_{n}^{2}\left(x_{0}\right)}{\left|B_{n}\right|}= \\
& =\frac{\sum_{x \in B_{n}} \gamma_{n}(x)}{\left|B_{n}\right|}+\frac{\left|B_{n} \cap I\right|}{\left|B_{n}\right|} \underset{n \rightarrow \infty}{\longrightarrow} \chi^{f}(p)+\theta(p) .
\end{aligned}
$$

С другой стороны,

$$
\begin{aligned}
\frac{\sum_{i=0}^{k(n)} \gamma_{n}^{2}\left(x_{i}\right)}{\left(\sum_{i=0}^{k(n)} \gamma_{n}\left(x_{i}\right)\right)^{2}} k(n) & =\frac{\sum_{i=1}^{k(n)} \gamma_{n}^{2}\left(x_{i}\right)+\left|B_{n} \cap I\right|}{\left(\sum_{i=1}^{k(n)} \gamma_{n}\left(x_{i}\right)+\sqrt{\left|B_{n} \cap I\right|}\right)^{2}} k(n) \leqslant \\
& \leqslant \frac{\sum_{x \in B_{n}} \gamma_{n}(x)+\left|B_{n} \cap I\right|}{\left(\sum_{i=1}^{k(n)} \gamma_{n}\left(x_{i}\right)\right)^{2}} k(n)=\frac{\sum_{x \in B_{n}} \gamma_{n}(x)+\left|B_{n} \cap I\right|}{\left|B_{n} \backslash I\right|^{2}}= \\
& =\frac{\left|B_{n}\right|^{2}}{\left|B_{n} \backslash I\right|^{2}} \frac{\sum_{x \in B_{n}} \gamma_{n}(x)+\left|B_{n} \cap I\right|}{\left|B_{n}\right|} \frac{k(n)}{\left|B_{n}\right|} \underset{n \rightarrow \infty}{\longrightarrow} \frac{\chi^{f}(p)+\theta(p)}{(1-\theta(p))^{2}} \kappa(p), \\
\frac{\sum_{i=0}^{k(n)} \gamma_{n}^{2}\left(x_{i}\right)}{\left(\sum_{i=0}^{k(n)} \gamma_{n}\left(x_{i}\right)\right)^{2}} k(n) & =\frac{\sum_{i=1}^{k(n)} \gamma_{n}^{2}\left(x_{i}\right)+\left|B_{n} \cap I\right|}{\left(\left|B_{n} \backslash I\right|+\sqrt{\left|B_{n} \cap I\right|}\right)^{2}} k(n) \geqslant \\
& \geqslant \frac{\sum_{i=1}^{k(n)} \gamma_{n}^{2}\left(x_{i}\right)}{\left(\left|B_{n} \backslash I\right|+\left|B_{n} \cap I\right|\right)^{2}} k(n)= \\
& =\frac{\sum_{x \in B_{n}} \gamma_{n}(x)}{\left|B_{n}\right|^{2}} k(n) \underset{n \rightarrow \infty}{\longrightarrow} \chi^{f}(p) \kappa(p) .
\end{aligned}
$$

Отсюда получаем

$$
\frac{\sum_{i=0}^{k(n)} \gamma_{n}^{2}\left(x_{i}\right)}{\left(\sum_{i=0}^{k(n)} \gamma_{n}\left(x_{i}\right)\right)^{2}}=O\left(\frac{1}{k(n)}\right),
$$

и второе утверждение теоремы вытекает теперь из п. 2 предложения.

ДокАЗАТЕЛьство тЕорЕмы 4. Центральная предельная теорема для annealedвероятности следует из теоремы 3 на основании рассуждений, аналогичных тем, что были приведены при доказательстве теоремы 2.

Благодарности. Авторы благодарны рецензенту за тщательное изучение работы и многочисленные ценные предложения. Работа частично поддержана фондом Natural Science Foundation of China (гранты № 10531070, 10371074). 


\section{Список литературы}

[1] M. T. Barlow, Ann. Probab., 32:4 (2004), 3024-3084.

[2] N. Berger, M. Biskup, Probab. Theory Related Fields, 137:1-2 (2007), 83-120.

[3] C. Hoffman, E. Mossel, Potential Anal., 14:4 (2001), 375-385.

[4] P. Mathieu, E. Remy, Ann. Probab., 32:1A (2004), 100-128.

[5] O. Häggström, Stochastic Process. Appl., 96:2 (2001), 213-242.

[6] M. Kaufman, J.E. Touma, Phys. Rev. B, 49:14 (1994), 9583-9585.

[7] C. L. Henley, Phys. Rev. Lett., 54:18 (1985), 2030-2033.

[8] O. Häggström, Ann. Appl. Probab., 9:4 (1999), 1149-1159.

[9] O. Garet, ESAIM Probab. Stat., 5 (2001), 105-118 (electronic).

[10] G. Grimmett, Percolation, Springer, Berlin, 1989.

[11] S. Havlin, R. Nossal, J. Phys. A, 17:8 (1984), L427-L432.

[12] C. M. Newman, L. S. Schulman, J. Stat. Phys., 26:3 (1981), 613-628.

[13] H. Kesten, "Some highlights of percolation", Proc. Internat. Congr. of Mathematicians, V. 1, Higher Education Press, Beijing, 2002, 345-362.

[14] А. А. Темпельман, Труды MMO, 26 (1972), 95-132.

[15] M. Penrose, Random Geometric Graphs, Oxford Stud. Probab., 5, Oxford University Press, Oxford, 2003.

Поступила в редакцию 13.11.2007, после доработки 1.02.2008 